\title{
LINTERNAS EN LA NOCHE. UNA PRIMERA APROXIMACIÓN A LAS METODOLOGÍAS DE ACOMPAÑAMIENTO INTENSIVO PARA PROYECTOS CULTURALES
}

Lanterns in the night. A first approximation to intensive accompaniment methodologies for cultural projects

\section{Roberto Ramos de León \\ Multilateral, Asociación Aragonesa para la \\ Cooperación Cultural, España \\ robertoramos@multilateral.info}

RESUMEN: El artículo trata de explicar cómo la necesidad de incorporar y/o sostener estrategias de acompañamiento profesional a proyectos culturales constituye una de las vías más plausibles para el acceso al mercado laboral. Se aborda un marco teórico y metodológico, basado en publicaciones especializadas, así como en la propia experiencia del Centro de documentación Cultura y Empleo, Infoculture, promovido por la Asociación Multilateral y que, en la ciudad de Huesca (España), ha venido avanzando en este tipo de procesos que actualmente se están extendiendo en diferentes instituciones culturales, públicas y privadas.

Palabras clave: acompañamiento cultural, gestión de información cultural, asesoramiento, profesionalización, empleo cultural

RESUM: L'article tracta d'explicar com la necessitat d'incorporar i/o sostindre estratègies d'acompanyament professional a projectes culturals constitueix una de les vies més plausibles per a l'accés al mercat laboral. S'aborda un marc teòric i metodològic, basat en publicacions especialitzades, així com en la pròpia experiència del Centre de documentació Cultura i Ocupació, Infoculture, promogut per l'Associació Multilateral i que, a la ciutat d'Osca (Espanya), ha vingut avançant en aquesta mena de processos que actualment s'estan estenent en diferents institucions culturals, públiques i privades. 
Paraules clau: acompanyament cultural, gestió d'informació cultural, assessorament, professionalització, ocupació cultural.

ABSTRACT: The article explains the importance of introducing and/or supporting professional counselling strategies for cultural projects as an essential way for joining the labour market. It addresses a theoretical and methodological framework based on specialized publications and the Documentation Centre about Culture and Employment (Huesca, Spain) own experience, promoted by the Association Multilateral. Nowadays, public and private cultural organizations are incorporating these methodologies in their work processes.

KeYWORDS: cultural professional counselling, cultural information management, assessment, professionalization, cultural employment

\section{El acompañamiento cultural en su contexto}

i

Merece el sector cultural un apoyo singular en materia de empleo? Esta duda ronda en la cabeza de quienes no conocen en profundidad las circunstancias laborales en las que se desenvuelven los profesionales de las artes y de la cultura.

Si bien existen diferencias notables entre perfiles y entre disciplinas, también son conocidas las dificultades que, en general, se encuentran aquellas personas que deciden convertir su vocación artística y cultural en profesión.

Este artículo pretende abordar, desde un prisma general, los procesos de acompañamiento para el empleo cultural: cómo surgen, sus características y referentes, teniendo en cuenta la propia experiencia y trayectoria de Multilateral en esta materia desde el año 2002. 
Como señala el investigador cultural Jaron Rowan, en la década de los años noventa del pasado siglo, varios estados profundizan en las relaciones entre cultura y economía. En su libro Emprendizajes en cultura, el autor señala cómo, en 1994, el gobierno australiano «intentará redefinir el potencial y tamaño del sector cultural a través de un documento denominado 'Creative Nation: Commonwealth Cultural Policy'». ${ }^{1}$ Este informe subraya la importancia «del valor estratégico de las industrias culturales como motor de crecimiento» e indica que «El Gobierno ayudará a las organizaciones culturales para que operen de forma más efectiva dentro de un marco económico a través de programas de asistencia industrial que incluirán las industrias culturales». ${ }^{2}$ El documento merece una especial atención al centrar su interés en el apoyo profesional directo a proyectos empresariales de agentes culturales y no únicamente a otras instituciones o programas públicos.

En el camino del emprendizaje cultural, Rowan cita al gobierno laborista de Tony Blair, que pone el acento en las políticas culturales públicas y, con ellas, un apoyo más intensivo al sector cultural y creativo: se realizan informes, prospecciones y mapeos sobre la situación y sus capacidades, además de poner en marcha «organismos como el Creative Industries Task Force $[. .$.$] que nacieron con el objetivo de [...] estimular la generación de$ riqueza y empleo a través de las industrias creativas» ${ }^{3}$.

La acción política ha buscado, en estos casos, mejorar las perspectivas laborales de los artistas e incluir este sector dentro de dinámicas empresariales con una visión más amplia pues, como se indicaba anteriormente, la casuística de los profesionales es extensa. Como señala la economista Françoise Benhamou, «las decisiones sobre los mercados de trabajo desempeñan un importante papel en la cuestión fundamental de la selección del talento» ${ }^{4}$. Por ello, ante la fragilidad o debilidad frente al mercado, deben desarrollarse políticas laborales orientadas al empleo cultural.

1. Rowan, J. (2010). Emprendizajes en cultura. Traficantes de sueños.

2. Rowan, J. (2010). Emprendizajes en cultura. Traficantes de sueños.

3. Rowan, J. (2010). Emprendizajes en cultura. Traficantes de sueños.

4. Benhamou, F. (2005). Los mercados de trabajo de los artistas. En Ruth Towse. Manual de economía de la cultura. Datautor. 
En este marco, las medidas tienden al proteccionismo del sector en general, a la captación de recursos o al desarrollo formativo. Algunos ejemplos de iniciativas destacables los constituyen medidas de apoyo como el estatus de intermitencia o la prestación del servicio cívico en organizaciones culturales (Francia), incentivos al patrocinio cultural mediante desgravaciones o sistemas de loterías (Reino Unido); programas de apoyo profesional al sector cultural en casi todos los países; o respeto a la propiedad intelectual. Además, en la mayoría de los países de nuestro entorno se promueve el consumo cultural mediante programas de mediación, desarrollo de audiencias, reducción de impuestos indirectos o estatutos laborales diferenciados.

Para conocer el contexto en España en los albores de la profesionalización del sector, resulta útil referirse a instituciones que desde hace décadas han venido trabajando en esta línea. Entre todas ellas, merece un lugar destacado el Centre d'Estudis i Recursos Culturals ${ }^{5}$ (CERC) de la Diputación de Barcelona, fundado por Eduard Delgado, que a mediados de la década de los años ochenta, ofrece un papel visionario sobre la conciencia de sector y la necesidad de apoyo a sus profesionales.

Asimismo, cabe destacar algunos actores que han impulsado el estudio sobre las relaciones entre cultura y economía y sus aportaciones al tejido socioeconómico. Un papel relevante debe atribuirse a las investigaciones del Departamento de Economía Aplicada de la Universitat de València y, más concretamente, a la Unidad de Investigación ECONCULT, ${ }^{6}$ dirigida por Pau Rausell. En este campo, el Observatorio Cultural del Proyecto Atalaya ${ }^{7}$ de las universidades andaluzas, o el Instituto de Estudios de Ocio de la Universidad de Deusto ${ }^{8}$, han desarrollado líneas de investigación y trabajos muy útiles. Académicamente, los estudios de gestión cultural o cooperación cultural de las universidades de Barcelona, Zaragoza, Girona o la Complutense, entre otras, han contribuido a la profesionalización.

5. https://www.diba.cat/es/web/cerc [Consultado en septiembre de 2019]

6. http://www.econcult.eu/es/ [Consultado en septiembre de 2019]

7. https://www.observatorioatalaya.es [Consultado en septiembre de 2019]

8. https://socialesyhumanas.deusto.es/cs/Satellite/socialesyhumanas/es/institutoestudios-ocio [Consultado en septiembre de 2019] 
Del fuerte interés por investigar y desarrollar las relaciones de los agentes culturales con el plano laboral, surge un manual sobre creación de empresas culturales dirigido por José Ruiz Navarro en 2008. ${ }^{9}$ En él se constata la necesidad de que «la formación específica en creación y gestión empresarial ayudaría a mejorar la tasa de creación y supervivencia de las empresas culturales». Y continúa, «en el tema de la formación, las empresas atribuyen una gran importancia a la formación específica en gestión cultural, la cual, señalan, favorece la creación de empresas culturales en mayor medida que el conocimiento en gestión de empresas, aunque ambas se complementan». ${ }^{10}$

Desde el tercer sector, Interarts ${ }^{11}$ en Barcelona y Multilateral ${ }^{12}$ en Huesca han venido trabajando desde los años noventa en proyectos ligados a la movilidad profesional, la cooperación cultural internacional y los servicios de información, asesoramiento y acompañamiento cultural.

Precisamente Interarts publica en 2011 un informe sobre sistemas de información para la movilidad cultural en España, ${ }^{13}$ dentro de su proyecto Practics. Este documento surge como consecuencia de un encuentro entre servicios de información y asesoramiento cultural en España y en él se concluye sobre la necesidad de extender «seminarios de formación para profesionales capaces de multiplicar la información existente y acompañar a otros artistas y profesionales de la cultura en procesos de internacionalización». El documento retrata la relevancia asumida por servicios que ejercen un papel multiplicador en la difusión de información profesional, la cual acaba derivando en nuevas oportunidades de empleo.

Multilateral, por su parte, en el marco del proyecto europeo Convivencia Pirineos Mediterráneo analiza el caso de los dispositivos de asesoramiento y acompañamiento en dinámicas transfronterizas, constatando la necesidad de

9. Ruiz Navarro, J. (dir.). (2008). Creación de empresas en el ámbito cultural. Fundación Autor.

10. Ruiz Navarro, J. (dir.). (2008). Creación de empresas en el ámbito cultural. Fundación Autor.

11. https://www.interarts.net/?lang=es [Consultado en septiembre de 2019]

12. http://www.multilateral.info [Consultado en septiembre de 2019]

13. Fundació Interarts (2011). Sistemas de información sobre la movilidad cultural en España: diagnóstico y prospectiva [en línea]. Disponible en: http://www.interarts. net/descargas/interarts944.pdf [Consultado en septiembre de 2019] 
crear «ventanillas únicas para profesionales del sector en materia de ayudas e internacionalización». ${ }^{14}$

Pero la idea de acompañamiento también se consolida en otros estudios, itinerarios formativos y programas de innovación empresarial. No obstante, el análisis de esta materia que se relata en las páginas siguientes se basa en otro modelo, en un concepto de servicio de acompañamiento con un espacio físico, accesible para agentes no ligados estrictamente a formaciones.

La clave radica en que un servicio de acompañamiento persigue en última instancia la sostenibilidad de los proyectos culturales. Se trata de una inversión que retroalimenta al propio sector cultural, a la sociedad y al tejido económico en el que se asienta. Como señala el economista David Throsby «descuidar el capital cultural, permitiendo que el patrimonio se deteriore, no apoyando los valores culturales que proporcionan a las personas un sentido de identidad, no adoptando la inversión necesaria para mantener o aumentar el stock de capital cultural tangible o intangible (por ejemplo, mediante la producción de obras de arte), también pondrá en peligro los sistemas culturales, y puede hacer que se derrumben, con la consiguiente pérdida de bienestar y producción $»^{15}$. Por ello, más allá del beneficio que representa el impulso a la creación cultural y el estudio del patrimonio cultural, se precisan servicios que trabajen en apoyar esa sostenibilidad económica.

Lo contrario sería dejar al azar la financiación entre el «sacrificio personal, el apoyo familiar, los mecenas, el Estado y el mercado» ${ }^{16}$, como indica el intelectual mexicano Gabriel Zaid en Dinero para la cultura. Todas esas vías «pueden liberar o esclavizar» a los creadores y «todas tienen consecuencias en la obra, más allá de sus efectos financieros» ${ }^{17}$. Circular en la dirección equivocada supondría dirigirse hacia el estancamiento o el abandono de proyectos y vocaciones por falta de apoyo informativo y formativo, con un destino inexorable hacia el mainstream.

14. Raimondi, J. (2011). Miradas al desarrollo del territorio transfronterizo pirenaico: hacia una cooperación cultural más eficiente. Proyecto Convivencia Pirineos Mediterráneo.

15. Throsby, D. (2005). La sostenibilidad cultural. En: Towse, R. Manual de economía de la cultura. Datautor.

16. Zaid, G. (2013). Dinero para la cultura. Debate.

17. Zaid, G. (2013). Dinero para la cultura. Debate. 
Entonces, volviendo a la pregunta inicial, ¿merece el sector cultural un apoyo singular en materia de empleo? Sin duda, porque el propio sector ya presenta una singular idiosincrasia y porque resulta obvio que no apoyar a un sector frágil, pero rico y espléndido con la sociedad, equivale aquí a empobrecer a todos: «En una sociedad gobernada por la mayoría, como aseguras que es, la Democracia es Democracia si su empresa es defender los derechos del más oscuro volumen de poesía». ${ }^{18}$

\section{Un camino hacia el acompañamiento profesional en cultura}

En el caso de la asociación Multilateral, ${ }^{19}$ con sede en Huesca (España), en el año 2002 se pone en marcha el proyecto transfronterizo Espacio Infoculture, junto a la entidad Cépière Formation de Toulouse (Francia). Una iniciativa que se hace realidad a través de fondos europeos, y de instituciones locales y regionales interesadas en apoyar la finalidad del proyecto: la profesionalización de agentes culturales y la promoción de iniciativas culturales en el Pirineo Central.

La cristalización de aquel proyecto inicial fue el Centro de documentación Cultura y Empleo, Infoculture. Desde entonces, el proyecto ha atravesado diversos estadios que le han permitido avanzar en el conocimiento e investigación de metodologías de apoyo al empleo cultural. En 2016 contaba con cerca de 10.000 personas asociadas y su sitio web (www.infoculture.info) era uno de los más reconocidos en su ámbito.

Una de las líneas de actuación que demostró una mayor utilidad fue la relativa al acompañamiento profesional a cuyas características se refiere este artículo.

18. Themerson, S. (1951). Wooff, Wooff or Who Killed Richard Wagner? Gaberbocchus Press.

19. http://www.multilateral.info [Consultado en septiembre de 2019] 


\section{Otras maneras de hacer para un entorno que aprieta}

La crisis de 2008 representó en España un antes y un después en la tendencia del mercado laboral, cuando el sector cultural fue especialmente afectado, entre otras razones, por su fuerte dependencia de la Administración, obligada a cumplir con objetivos de déficit público, traducidos en recortes presupuestarios.

Las consecuencias en un centro sobre «Cultura y Empleo» supusieron la multiplicación de la demanda en forma de suscripciones a las alertas de empleo, consultas y citas. Fue necesario profundizar entonces en otras metodologías para encarar el enorme reto: abordar el derrumbe del mercado laboral en el sector. No obstante, entre los años 2008 y 2011 confluyeron dos circunstancias que permitieron al centro afrontar mejor la atención a los agentes culturales.

Por un lado, la Administración fue tomando conciencia de la virulenta situación a la que se enfrentaba el sector privado y que, poco después, acabaría engullendo algunos de los servicios, e incluso organismos, dependientes de ella. El Departamento de Educación, Cultura y Deporte del Gobierno de Aragón acordó suscribir un convenio con la Asociación Multilateral (promotora del centro) para que pudiera prestar unos servicios que se iban desbordando por la fuerte demanda, y cuya extensión llegó hasta 2011. Una vez finalizado, el Ayuntamiento de Huesca apostó por mantener y promover el centro en consonancia con las posibilidades y recursos de una entidad local. Por último, el Ministerio de Educación, Cultura y Deporte, subvencionó a través de tres convocatorias la mejora de los servicios sobre empleo y emprendimiento cultural que se prestaban.

La segunda circunstancia, por otro lado, fue la siguiente. Entre 2009 y 2011, la asociación Multilateral participó como socia en un proyecto europeo de cooperación cultural transfronteriza con otras entidades de Cataluña y de las regiones francesas de Languedoc-Roussillon y Midi-Pyrénées (hoy fusionadas como Occitania). Este proyecto, denominado Convivencia Pirineos-Mediterráneo, permitió contratar personal para la ejecución de sus acciones, ligadas a la capacitación, profesionalización e internacionalización de agentes culturales. 
No es baladí, por tanto, que en dicha coyuntura el centro tuviera capacidad para hacer permeables y ejecutar muchas buenas prácticas (entre ellas el acompañamiento) cuya utilidad ya se había constatado en el proyecto Espacio Infoculture. Convivencia Pirineos Mediterráneo resultó fundamental esta ejecución, puesto que decidió crear una Célula de Ingeniería Cultural con tres niveles derivados de las necesidades profesionales:

A. Nivel de información, caracterizado por respuestas a cuestiones objetivas, simples, únicas y, generalmente, urgentes.

B. Nivel de asesoramiento, que se corresponde con demandas informativas con mayor grado de elaboración por parte del servicio y que pueden precisar, en algunos casos, de acciones subjetivas. La diferenciación respecto al nivel anterior es fundamentalmente la mayor implicación del servicio, pues necesitan su consejo, o su derivación a otros agentes relacionados. La resolución de la demanda puede ser inmediata o no, dependiendo del nivel de conocimiento de la cuestión.

C. Nivel de acompañamiento, sobre el que trata este artículo, y que cuenta con una mayor complejidad derivada de la subjetividad de cada proyecto profesional. Por buscar un símil, el acompañamiento no es una prenda prêt à porter, sino que responde más bien a la imagen de una sastrería en la que se toman medidas, se encargan los detalles si no se encuentran en tienda y, si fuera el caso, se reajusta el traje o vestido una y otra vez. Capacidades, necesidades, espacio y tiempo son cuatro de los elementos que van a delimitar el éxito de un proceso de este tipo. 


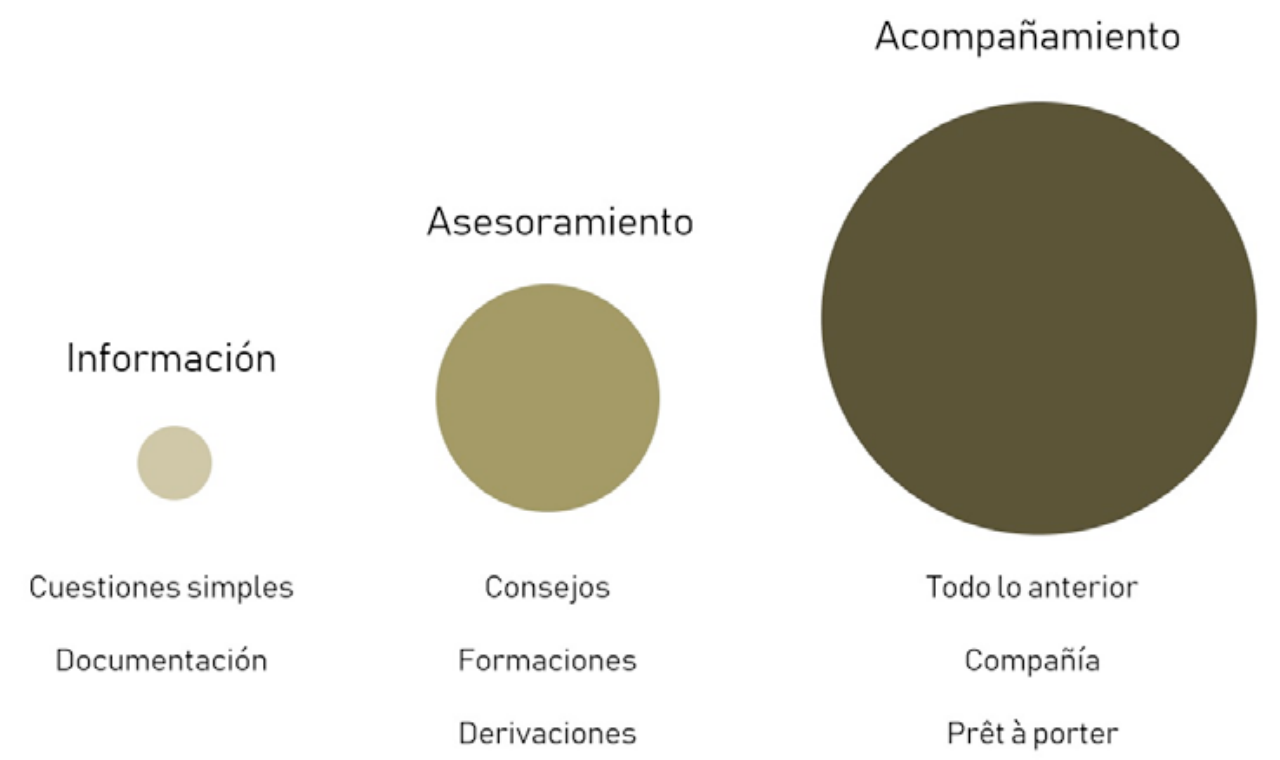

Figura 1. Niveles de apoyo para el empleo a agentes culturales.

Cabe señalar que el centro de Huesca se ha apoyado en otros recursos formativos o de documentación con los que cuenta, unos 2.000 volúmenes.

Finalmente, el Ayuntamiento de Huesca decide crear, en 2016, un servicio de información y acompañamiento para el fomento del empleo cultural, como herramienta complementaria a un nuevo coworking cultural municipal en la ciudad. Desde entonces, este servicio municipal se ha encargado de apoyar a los agentes culturales instalados en este equipamiento a través de acciones de información, asesoramiento y acompañamiento, además de formaciones.

También, a finales de 2017, el Ayuntamiento de Zaragoza crea el servicio Zaragoza Cultura Acompaña, ${ }^{20}$ ligado igualmente a la mejora de las capacidades profesionales de los agentes culturales de la ciudad, gestionado por Multilateral. Este servicio realiza labores de información, documentación, asesoramiento y acompañamiento, basados principalmente en el modelo de «ciclo del proyecto cultural» y la creación de valor en sus fases. El servicio ha realizado una importante labor de prospección de la realidad del tejido cultural zaragozano.

20. http://Www.zcacompana.com [consultado en septiembre de 2019] 


\section{El servicio de acompañamiento a proyectos culturales}

Para empezar, resulta necesario aclarar que este apartado hace referencia a un acompañamiento destinado a agentes culturales profesionales o en vías de profesionalización que deseen hacer del arte y de la cultura su medio de vida.

Un servicio de acompañamiento cultural encierra un cierto grado de complejidad puesto que conocer a fondo cada disciplina y género resulta una tarea inconmensurable. Por ello, parte de la base del realismo en las intervenciones, desde la honestidad de lo que se conoce y de lo que no. Al fin y al cabo, el agente acompañante también aprende en un proceso «simbiótico».

Esta concepción parece fundamental y permite definir el verbo acompañar a efectos del servicio: «ir junto a alguien hacia el mismo lugar y en el mismo momento». A partir de aquí, la experiencia sobre el terreno ha demostrado que el proceso de acompañar se asimila principalmente a tres verbos: guiar, escoltar, conducir:

- Guiar, en tanto en cuanto el acompañante debe abrir el horizonte al acompañado, le muestra el camino hacia un empleo cultural. Ejemplos de ello son los consejos sobre el régimen jurídico de la estructura o vías para la facturación.

- Escoltar, en el sentido de tratar de anticiparse a los riesgos que suceden en el itinerario, tales como propiciar el conocimiento al agente acompañado sobre una convocatoria que podría no publicarse o la inadecuación de su perfil profesional a los nuevos retos.

- Conducir, reservado únicamente en aquellos casos en los que, ante un proyecto aparentemente viable y sostenible, se presenta una crisis de estructura o existe una carencia de habilidades técnicas. Para estos casos, se analiza la situación y se apoya intensivamente durante el periodo de tiempo más breve posible, en aras a retornar al proceso de guía. Un ejemplo de conducción se produce cuando fallan las capacidades para comunicar un proyecto por desconocimiento de herramientas básicas. En este caso, el agente acompañante interviene para enseñárselas de forma dirigida. 
Por la experiencia de Multilateral en la aplicación de estas metodologías, la proporción ideal en el proceso de acompañamiento se establece en un $70 \%$ como guía, un $20 \%$ como escolta y un $10 \%$ como conductor del proyecto.

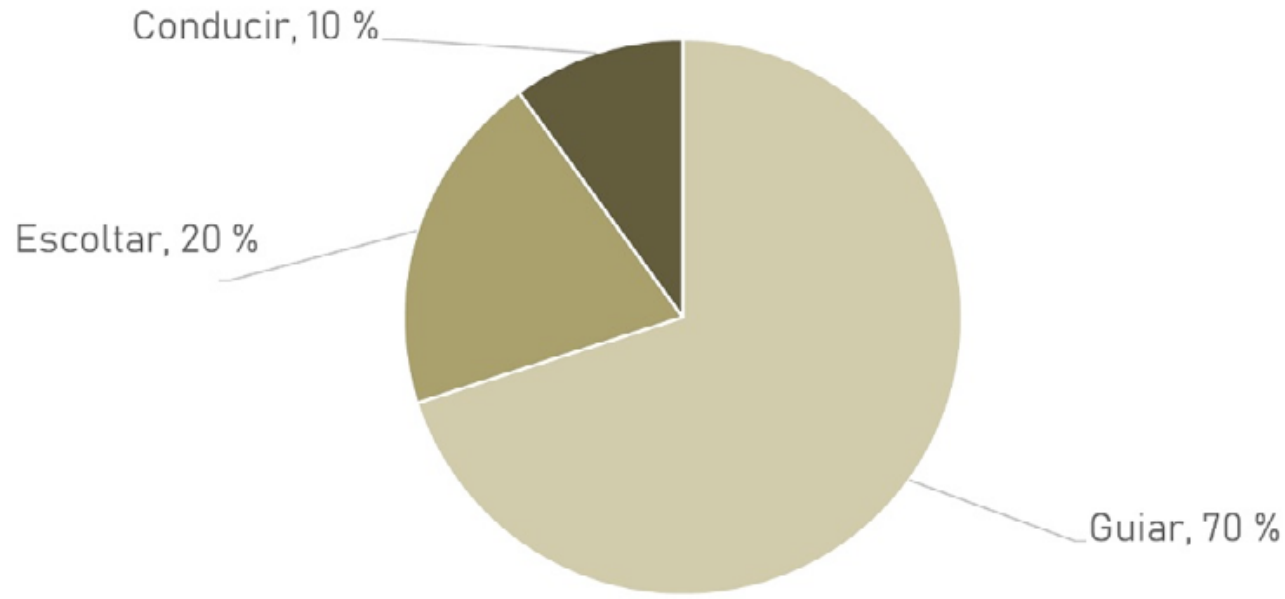

Figura 2. Conceptos sobre los que se derivan las acciones y tareas de un servicio de acompañamiento cultural.

\section{Fases de un proceso de acompañamiento}

A nivel práctico, la metodología de acompañamiento cuenta con los estadios siguientes:

1. Presentación de una iniciativa cultural (posible proyecto o entidad) por parte de un agente profesional o en vías de profesionalización. A priori, la idea general expuesta debe mostrar ya una mínima solvencia profesional y de viabilidad.

2. Aceptación por parte del servicio. En esta valoración, debe tenerse muy en cuenta que el acompañamiento precisa dedicación de tiempo (con su correspondiente coste). Por consiguiente, resulta indispensable calibrar si existe disponibilidad temporal real para atenderlo adecuadamente. Para garantizar el éxito, no es recomendable acompañar simultáneamente más de diez proyectos por parte del mismo agente. Por ello, antes de comenzar un acompañamiento debe observarse el 
grado de compromiso del agente acompañado. En caso contrario, no se debería iniciar el proceso.

3. Conocimiento del otro, de su entorno, de su trayectoria, de su disciplina. La capacidad para «ponerse en la piel del otro» constituye el elemento clave del proceso. Una opinión muy habitual (y muy razonable) de quienes usan estos servicios consiste en que saben diferenciar muy bien cuándo se les toma en serio. En esta fase es importante generar empatía, cercanía, y también confianza a través de un código deontológico que preserve la privacidad.

4. Visualización de la viabilidad y sostenibilidad del proyecto como medio de vida a corto o medio plazo, dependiendo de la dificultad, capacidades, necesidades y urgencia del agente cultural. En el caso de que la iniciativa no parezca viable es mejor no continuar, para no generar falsas expectativas, o reorientarla siempre con la complicidad del agente promotor.

Para determinar la viabilidad y la sostenibilidad de un proyecto se aborda, en primer lugar, la estructura jurídica y administrativa del agente acompañado puesto que puede marcar a futuro las posibilidades de éxito del acompañamiento (posibilidades de convocatorias, facturación, etc.).

Dependiendo del proyecto, en esta fase resulta primordial trabajar en los ejes básicos que componen un proyecto cultural de carácter artístico: creación-gestión-mediación. Se trabaja aquí con diferentes herramientas como el benchmarking, el análisis DAFO, el modelo CANVAS o la reflexión e intervención sobre el ciclo cultural del proyecto.

5. Establecimiento de un calendario y de un espacio de trabajo en común, acorde con las posibilidades y capacidades reales. En el cronograma, que deberá dar como resultado una nueva entidad o un proyecto cultural nuevo o redefinido, se considera básico crear una dinámica acción-reacción. Por ello, se precisa ejecutar inmediatamente tras los encuentros en el servicio, puesto que un servicio de acompañamiento no pude permitir pérdidas de tiempo. 
Otro aspecto importante del acompañamiento que a menudo no se tiene en cuenta, es la idoneidad del espacio físico o virtual (por videoconferencia) en el que se produce: debe llevarse a cabo en una sala que permita la concentración, sin ruidos ni interferencias, y con un ordenador conectado a Internet.

6. Conexión con otros servicios y agentes (culturales o no). Durante el itinerario, en muchos casos, se observa la necesidad de trasladar el acompañamiento o hacerlo simultáneo con otros que se prestan desde áreas de desarrollo u otros agentes sociales y económicos. En casos de bloqueo se puede, además, proponer visitas profesionales a espacios que sirvan para abrir la mente o citas con agentes culturales que, desde su experiencia, pueden servir de apoyo.

7. Plasmación del proyecto cultural con los análisis previos de viabilidad, finalidad, objetivos, actividades, calendario, presupuesto e indicadores que deben cumplirse. El documento de proyecto se complementa con otro gráfico y, ambos, pueden presentarse ante terceros y/o constituir un cuaderno de navegación imprescindible para la propia entidad o proyecto.

En esta fase se pueden aplicar diferentes metodologías para el diseño de proyectos culturales, siendo una de las más utilizadas en el sector la estructurada por David Roselló.21 En su definición, debe insistirse en la elaboración de planes de financiación, comunicación y desarrollo de audiencias, así como la evaluación de los proyectos a través de indicadores.

8. Finalización del proceso. El mecanismo de apoyo llegará a buen fin si ha sido capaz de poner en marcha un proyecto cultural que permita al agente cultural vivir de su profesión. Por la experiencia de Multilateral, existen casos de éxito en un tiempo razonable y otros que, debido a las circunstancias cambiantes, se reorientan para que fructifiquen, o terminan abandonándose.

21. Roselló, D. (2007). Diseño y evaluación de proyectos culturales. Ariel. 
En todos estos casos, la riqueza del acompañamiento es mutua para acompañante y acompañado pero, sobre todo, el propio agente cultural será capaz de atreverse en el futuro a crear su proyecto, usando el servicio solamente para información o asesoramiento.

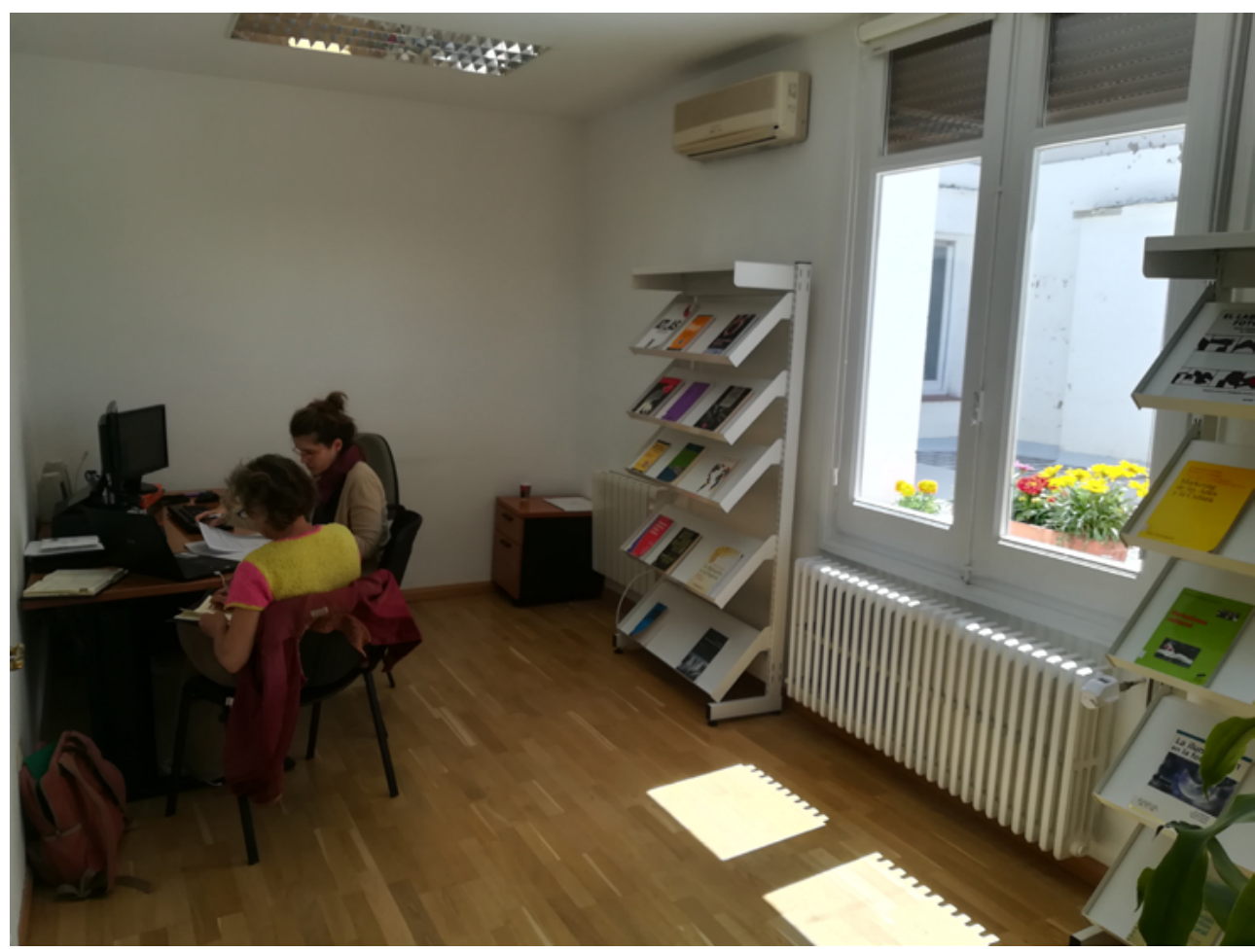

Figura 3. Reunión derivada del servicio de acompañamiento entre dos entidades (Zerclo Patrimonio Sostenible y Muretes de Arte), ambas acompañadas desde el Servicio de Información y Acompañamiento del Ayuntamiento de Huesca.

\section{Crear un servicio de acompañamiento cultural}

Lógicamente, poner en marcha un servicio de acompañamiento dependerá en gran medida del tipo de organización.

Si depende de una comunidad autónoma, será necesaria la creación de una oficina dotada con personal especializado tanto en gestión de información y documentación cultural, así como en gestión y políticas culturales. Esto permitirá una mayor fluidez para el acompañamiento porque mientras el perfil de documentalista controla las convocatorias, informes y estadísticas culturales, documentación básica y difunde información, la persona con un perfil 
de gestión cultural complementa y aplica la información a la metodología de acompañamiento y dota a los proyectos de alto valor añadido. En una propuesta de máximos, el servicio debería crear perfiles profesionales de acompañamiento para cada disciplina.

Para el éxito del servicio debería contar con una marca identificable, un sitio web autónomo, un sistema DSI (difusión selectiva de la información) para el envío de alertas a la carta, un boletín digital y un centro de documentación solvente. Según los intereses de la Administración, se crearán perfiles diferenciados en redes sociales, que diferencien público de profesionales.

Algunos ejemplos de servicios de asesoramiento y acompañamiento $\mathrm{y} / \mathrm{o}$ formación a escala autonómica son el Proyecto Lunar de Andalucía Emprende, Fundación Pública Andaluza ${ }^{22}$ o el Servei de Desenvolupament Empresarial $(\mathrm{SDE})$, del Institut Català de les Empreses Culturals, ${ }^{23}$ si bien la mayoría de comunidades autónomas cuentan o han contado con algún tipo de programa de apoyo empresarial, que incluye generalmente una mentorización, aunque sin servicio de acompañamiento permanente. Este es, por ejemplo, el caso del Programa de Emprendimiento de Industrias Culturales y Creativas ${ }^{24}$ del Instituto Aragonés de Fomento.

Cabe señalar que en el País Vasco se publicó un informe sobre acompañamiento público a las empresas culturales, donde se afirma que «el acompañamiento requiere también una responsabilidad por parte de la Administración, ya que supone una implicación continuada en el proceso de desarrollo de las empresas culturales y creativas a las que ofrece apoyo». ${ }^{25}$

22. http://www.proyectolunar.com/ [Consultado en septiembre de 2019].

23. https://sde.cultura.gencat.cat/cultura/que-fem-eapro_3 2.html [Consultado en septiembre de 2019]

24. https://www.emprenderenaragon.es/paginas/programa-de-emprendimiento-enindustrias-creativas-y-culturales [Consultado en septiembre de 2019]

25. Observatorio Vasco de la Cultura, Gobierno Vasco (2014). El acompañamiento público a las empresas culturales: análisis de casos y propuestas de actuación [en línea]. Disponible en: http://www.kultura.ejgv.euskadi.eus/contenidos/informacion/ keb argit laguntz publiko 2014/es def/adjuntos/Acompanamiento publico empresas culturales.pdf [Consultado en septiembre de 2019] 
En la Comunidad Valenciana, dentro del Plan Estratégico Cultural Valenciano 2016-2020, denominado comúnmente Fes Cultura, ${ }^{26}$ se aboga por la creación de diferentes oficinas de apoyo al sector (para proyectos europeos o para el desarrollo de audiencias y didáctica de las artes escénicas), si bien no utiliza el término acompañamiento.

En las administraciones públicas locales (diputaciones o cabildos, comarcas, ayuntamientos de ciudades), los recursos destinados dependen fundamentalmente de sus dimensiones, así como de la importancia que se otorgue a las políticas culturales como motor de desarrollo local. El asesoramiento puede reforzar el nivel municipal desde las mismas diputaciones, como es el caso del ya citado Centre d'Estudis i Recursos Culturals (CERC) de la Diputació de Barcelona, ${ }^{27}$ que desde 1986 trabaja en la formación, información y asesoramiento a los ayuntamientos de la provincia de Barcelona en materia de gestión y políticas culturales y que constituye todo un referente de ámbito nacional e internacional. Su centro de documentación se ha erigido como una fuente de investigación sobre políticas, programas y proyectos culturales, a la par que ha impulsado la profesión mediante eventos formativos y encuentros bienales como Interacció. ${ }^{28}$

En cuanto a comarcas y ayuntamientos, como administraciones más cercanas a la ciudadanía y ámbito territorial menos extenso, resulta más sencillo prestar un apoyo directo a los agentes culturales. Como se ha indicado, al abordar los antecedentes de este tipo de programas, además del Servicio de Información y Acompañamiento para el Empleo Cultural del Ayuntamiento de Huesca, dependiente de las áreas de Cultura y Desarrollo, el Ayuntamiento de Zaragoza puso en marcha a finales de 2017 el servicio Zaragoza Cultura Acompaña, ${ }^{29}$ dependiente de la sociedad municipal Zaragoza Cultural.

26. Generalitat Valenciana (2016). Fes Cultura. Plan Estratégico Cultural Valenciano 2016-2020 [en línea], Disponible en: http:/www.ceice.gva.es/ documents/161634256/162466274/FES+CULTURA+esp.pdf/c262f209-3503421c-a389-d7777c8009a3

27. https://www.diba.cat/es/web/cerc [Consultado en septiembre de 2019].

28. http://interaccio.diba.cat/ [Consultado en septiembre de 2019].

29. http://www.zcacompana.com [Consultado en septiembre de 2019]. 
Otros ayuntamientos, comarcas y diputaciones han creado o están creando oficinas de este tipo. Se citan algunos ejemplos como la Diputación de Álava, ${ }^{30}$ la Diputación de Guipúzcoa con Kbulegoa, ${ }^{31}$ el SARC de la Diputación de Valencia, ${ }^{32}$ el Ayuntamiento de Girona ${ }^{33}$ o la comarca de la Selva,${ }^{34}$ si bien la casuística puede distar mucho de unos servicios a otros.

Para municipios pequeños y medianos, sería importante que estos servicios de acompañamiento cultural pudieran prestarse a través de entidades supramunicipales o mediante una especialización de los agentes de desarrollo local (ADL).

En el ámbito académico, determinadas universidades como la Jaume I de Castelló, con su Programa de Extensión Universitaria en el territorio, ${ }^{35}$ la de Barcelona, con el Portal Iberoamericano de Gestión Cultural; ${ }^{36}$ o las universidades andaluzas con su proyecto Atalaya (especialmente el Observatorio de Políticas Culturales de la Universidad de Cádiz), ${ }^{37}$ llevan trabajando muchos años en procesos de información, formación, asesoramiento e incluso acompañamiento en diferentes campos.

En el sector privado pueden encontrarse también empresas o academias con programas de formación y acompañamiento, ligados a consultorías o incubadoras de proyecto como por ejemplo, Factoría Cultural. ${ }^{38}$ Son, a menudo,

30. https://www.kulturaraba.eus [Consultado en septiembre de 2019]

31. https://www.gipuzkoa.eus/es//-/kbulegoa [Consultado en septiembre de 2019]

32. https://www.sarc.es/va [Consultado en septiembre de 2019]

33. http://www2.girona.cat/ca/noticies/-/journal content/56 INSTANCE 9oNf $/ 11622 / 5913483$ ?refererPlid=24974 [Consultado en septiembre de 2019]

34. http://www.selvacultura.cat/ca/consultoria-de-cultura.html [Consultado en septiembre de 2019]

35. https://peu-uji.es/es/ [Consultado en septiembre de 2019]

36. http://www.ub.edu/cultural/portal-iberoamerica-de-gestio-cultural/?lang=es [Consultado en septiembre de 2019]

37. https://www.observatorioatalaya.es/ [Consultado en septiembre de 2019]

38. https://factoriacultural.es [Consultado en septiembre de 2019] 
servicios añadidos a coworkings tanto públicos como privados, como el caso de La Quinta del Sordo, en Madrid. ${ }^{39}$

Para finalizar, en el entorno europeo suelen proliferar los casos de asociaciones, fundaciones y redes culturales donde resulta habitual la prestación de estos servicios. Se citan a continuación algunos ejemplos próximos en el sur de Francia y norte de Italia:

- Occitanie en Scène. ${ }^{40}$ Situada en Montpellier, esta asociación cuenta con un dispositivo de ayuda a la difusión para compañías de artes escénicas de la región de Occitania.

- Unité Culture de l'Unión Cépière Robert Monnier. ${ }^{41}$ Se trata de una asociación de interés general con sede en Toulouse, que efectúa un acompañamiento integral a proyectos musicales, escénicos y visuales.

- Fondazione Cariplo. ${ }^{42}$ Entidad filantrópica con base en Milán cuya misión consiste en el sostenimiento, la promoción y la innovación de proyectos con utilidad social ligados en gran parte a la cultura.

- Proyecto Performing $+{ }^{43}$ Se trata de una iniciativa de artes escénicas en la región de Piamonte, creada por la Compagnia di San Paolo y la Fondazione Piemonte dal Vivo, con la colaboración del Observatorio de la Cultura de Piamonte. Ofrece un itinerario para agentes de diferentes entidades de la región que trabajan en el ámbito de las artes escénicas. Su duración es de tres años (2018-2020).

39. $\quad$ https://quintadelsordo.com/programa-de-acompanamiento-de-proyectosculturales/ [Consultado en septiembre de 2019]

40. https://www.reseauenscene.fr/dispositifs-accompagnement.html [Consultado en septiembre de 2019]

41. https://ucrm.fr/accompagnement-specifique-dans-le-domaine-artistique/ [Consultado en septiembre de 2019]

42. http://www.fondazionecariplo.it [Consultado en septiembre de 2019]

43. http://www.performingplus.it [Consultado en septiembre de 2019] 


\section{Resultados y conclusiones}

Desde Multilateral, el estudio realizado con los datos del Centro de documentación Cultura y Empleo, Infoculture, de Huesca entre 2012 y 2016 muestra una atención superior a 1.900 consultas relacionadas específicamente con procesos de información, asesoramiento y acompañamiento, principalmente en el nivel local, provincial y autonómico. Un gran número de ellas (945) se refieren a asesoramientos y, por supuesto, en mucha menor medida a acompañamientos.

Tomando la metodología de acompañamiento en un periodo que permita evaluar los resultados, de los diez proyectos que el Centro de documentación Cultura y Empleo Infoculture acompañó en el año 2015, seis son, en 2019, una realidad sostenible o están a punto de serlo: un estudio de arte, una nueva empresa de gestión cultural, el impulso a tres proyectos musicales y un artista visual. Estas iniciativas serían imposibles sin el empeño por convertir una idea en proyecto y la confianza que los agentes culturales han depositado en el servicio.

A través de la iniciativa Acompaña, proyecto subvencionado por el Ministerio de Educación, Cultura y Deporte, hasta marzo de 2017, se acompañaron cuatro proyectos en el ámbito estatal en una vuelta de tuerca más, mediante aulas virtuales y jornadas técnicas de apoyo. Se trataba de una compañía de danza, una academia de rap, una empresa de accesibilidad cultural y un proyecto de galería. Los resultados fueron positivos en tres de los casos y llevan a pensar en la necesidad de estos refuerzos de manera más constante por parte de entidades públicas o privadas.

A falta de un análisis mucho más profundo, se observa en ambas muestras un éxito de la metodología de acompañamiento entre el 60 y el $75 \%$ de los casos.

Por ello, la conclusión a la que dirigen las técnicas de acompañamiento permite considerar que la puesta en marcha de servicios de este tipo a cualquier escala sirve para estimular el propio sector, el mercado laboral y, en consecuencia, una mejora social y económica del territorio al que sirven. Si resulta desafiante que el sector cultural encuentre su lugar en las sociedades del siglo XXI para que estas avancen, cabe preguntarse: ¿cómo hacerlo posible 
si no se crean puntos de apoyo material e inmaterial desde donde remarcar el papel crucial de artistas y de otros profesionales culturales?

$\mathrm{Y}$ es por esto, por lo que un servicio de acompañamiento quiere erigirse como uno de esos puntos de apoyo. O, como reza el título del artículo, esa linterna que alumbra el suelo mientras se atraviesa la oscuridad, que nos sirve de guía, que nos previene de caídas o daños. Una luz para ver un camino, para verse a sí mismo, para ser visto por otros.

\section{Referencias}

Benhamou, F. (2005). Los mercados de trabajo de los artistas. En Ruth Towse. Manual de economía de la cultura. Datautor.

Fundació Interarts (2011). Sistemas de información sobre la movilidad cultural en España: diagnóstico y prospectiva [en línea]. Disponible en: http://www.interarts.net/descargas/interarts944.pdf [Consultado en septiembre de 2019]

Generalitat Valenciana (2016). Fes Cultura. Plan Estratégico Cultural Valenciano 2016-2020 [en línea], Disponible en: http://www.ceice.gva. es/documents/161634256/162466274/FES+CULTURA+esp.pdf/c262f209-3503421c-a389-d7777c8009a3

Observatorio Vasco de la Cultura, Gobierno Vasco (2014). El acompañamiento público a las empresas culturales: análisis de casos y propuestas de actuación [en línea]. Disponible en: http://www.kultura. ejgv.euskadi.eus/contenidos/informacion/keb_argit laguntz_publiko_2014/es_def/ adjuntos/Acompanamiento_publico_empresas culturales.pdf [Consultado en septiembre de 2019]

RAImond, J. (2011). Miradas al desarrollo del territorio transfronterizo pirenaico: hacia una cooperación cultural más eficiente. Proyecto Convivencia Pirineos Mediterráneo.

Roselló, D. (2007). Diseño y evaluación de proyectos culturales. Ariel.

Rowan, J. (2010). Emprendizajes en cultura. Traficantes de sueños.

Ruiz Navarro, J. (dir.). (2008). Creación de empresas en el ámbito cultural. Fundación Autor.

Themerson, S. (1951). Wooff, Wooff or Who Killed Richard Wagner? Gaberbocchus Press. 
Throsby, D. (2005). La sostenibilidad cultural. En: Towse, R. Manual de economía de la cultura. Datautor.

ZAID, G. (2013). Dinero para la cultura. Debate.

Nota: Los sitios web citados o consultados, se encuentran referenciados en el cuerpo del documento, allá donde procede. 